\title{
Rutting prediction of a granular material for base layers of low traffic roads
}

\author{
I. Pérez ; J. Gallego ${ }^{+}$ \\ *Universidade da Coruña. E. T. S. I. Caminos, Campus de Elviña, 15071 A Coruña, Spain \\ +Universidad Politécnica de Madrid. E. T. S. I. Caminos, Profesor Aranguren, s/n, 28040 Madrid, Spain
}

\begin{abstract}
This paper analyses the permanent deformation performance of an unbound granular material for base layers of low traffic roads. The material has been subjected to repeated triaxial loads. The shakedown theory was used to classify the structural response of the unbound granular material to the applications of load cycles. Three models were fitted to express the cumulative permanent strain as a function of the number of load cycles. In general, the predictions of two models previously studied by other researchers proved to be good but in the long-term, they tended to underestimate the measured values. In contrast, a third new model -the sum of two well known models- offered excellent predictions, which in the long term did not tend to either underestimate or overestimate the measured values.
\end{abstract}

Keywords: Granular material; permanent deformation; models, rutting, low-traffic road. 


\title{
Rutting prediction of a granular material for base layers of low traffic roads
}

\author{
I. Pérez ; J. Gallego ${ }^{+}$ \\ *Universidade da Coruña. E. T. S. I. Caminos, Campus de Elviña, 15071 A Coruña, Spain \\ +Universidad Politécnica de Madrid. E. T. S. I. Caminos, Profesor Aranguren, s/n, 28040 Madrid, Spain
}

\section{Introduction}

Low-traffic roads are usually made with flexible pavements in which unbound granular materials provide the most important structural element. Accordingly, the structural design of these pavements requires the mathematical description of the permanent behaviour of unbound granular materials. The goal is to predict the development of rutting in granular base courses. The repeated load triaxial (RLT) permanent deformation test has been widely used to determine permanent deformation characteristics of granular materials. In the RLT test, under repeated load cycles the accumulation of permanent strain is a gradual process where each load application contributes to the accumulation of strain by a small increment. Therefore, the number of load cycles is an important factor to consider in the analysis of the long term behaviour of granular materials.

In this sense, Pérez et al [1] carried out several analyses related to permanent deformation. In their conclusion they pointed out that mathematical models that predicted permanent deformation as a function of load cycles presented certain deficiencies. To be precise, in the long term these models underestimated or overestimated the observed values. In this paper additional work was done to improve the predictions. The objective was to examine the applicability of a non-linear model depending on the number of load cycles to predict the development of rutting in granular base courses of low traffic roads. The model is not found in the technical literature and consists of the combination of two well known models: the Sweere model and the Wolff model.

\section{Background information}

\subsection{Permanent deformation behaviour using the shakedown concept}

The shakedown concept holds that the strain behaviour of unbound granular materials gradually stabilises as the number of load cycles increases, making it possible to define a limit value for the accumulation of permanent strain. This stabilisation is only achieved when the applied stresses are low, given that high stresses would result in the continuous increase of permanent strain and gradual deterioration $[2,3]$. This theory is very useful for describing the behaviour of unbound 
granular material under repeated load cycles. For example, the curves in Figure 1 [3] show the typical development of the permanent deformation of granular materials subjected to different stresses. When the curves are plotted on a log-log scale they may be separated into three distinctive phases. At the beginning, the samples compact an initial amount during the very first load cycles. Then they usually continue to compact gradually over many load cycles. Because this portion is plotted as a straight line on a log-log scale, it is also referred to as linear deformation. In this phase the material is considered to be in behavioural "range $A$ ". This linear deformation will continue indefinitely and the material does not reach failure. However, when a higher stress is applied the permanent deformation is more elevated. In this phase the material is in "range B", but with a greater number of load cycles, the material might enter into "range $C$ " behaviour and, finally, reach failure. Lastly, if the applied stress is even higher, the permanent strain accumulates rapidly in a low number of load cycles. In this condition the material will reach failure and rutting will take place very quickly. In this phase the material is in behavioural "range $C$ ".

\subsection{Permanent deformation models as a function of number of load cycles}

Sweere [4] modelled the relationship between the logarithm of the cumulative permanent axial strain and the logarithm of load repetitions (Equation 1, Table 1). In this model, $\varepsilon_{1 p}(\%)$ is the cumulative permanent axial strain after $N$ load cycles are attained by means of RLT tests, and $A$ and $B$ are non-linear regression parameters. Intercept $A$ represents the permanent strain at $N=1$ on a $\log$-log scale. Slope $B$ corresponds to the rate of change of the $\boldsymbol{\varepsilon}_{1 \mathrm{p}}(\%)$ logarithm as a function of the change in the logarithm of $N$. As can be seen, equation 1 predicts an infinite deformation for an infinite number of load cycles. Sweere [4] found a satisfactory linear relationship between the two factors on a log-log scale. However, Pérez et al [1] confirmed a satisfactory fit but observed that when the material is in "ranges $\boldsymbol{A}$ and $\boldsymbol{B}$ ", equation 1 tended to underestimate the measured values. Wolff et al [5] measured the accumulated permanent deformation produced in granular road bases using Heavy Vehicle Simulator (HVS) tests. They verified that for a large number of cycles $(N>$ $10^{6}$ ) the values predicted for equation 1 differed from the real values. Therefore, Wolff et al [5] suggested an improved model (Equation 2-Table1) with which they obtained a good fit. This model was also fitted by Theyse [6] with good results. Equation 2 predicts an infinite deformation for an 
infinite number of cycles, as well. Coefficients $m, A$ and $B$ are non-linear regression parameters and $e$ is the base of the natural logarithm. Equation 2 consists of a linear and exponential component. The exponential component rapidly decays with an increasing number of load cycles. Hence, the permanent deformation tends to form a straight line $(m \cdot N+A)$ at high numbers of load cycles. Parameters $m$ and $A$ are the slope and the intercept of the straight line (asymptote) respectively; $B$ is a constant controlling the bend of the curve. With regard to this model the fit found by Pérez et al [1] may be considered quite satisfactory but it was observed that when the material is in "ranges $\boldsymbol{A}$ and $\boldsymbol{B}$ ", equation 2 tended to overestimate the measured values.

Francken and Clauwaert [7] and Kaloush and Witczak [8] used a non-linear model composed of two components (Equation 3-Table1) to study the permanent deformation behaviour of asphalt mixtures subjected to RLT tests. The same model was also proposed by Huurman [9]. Later, Werkmeister [10] and Arnold [11] selected this model for the practical application of the shakedown concept to model the permanent deformation of unbound granular materials. In equation 3, the first component is the same power-law proposed by Sweere. According to Werkmeister [10], it is able to express the material phase in behavioural "range A". The parameters $A_{1}$ and $B_{1}$ are similar to parameters $A$ and $B$ of equation 1 . The second component is a function which represents an exponential increase of $\varepsilon_{1 \mathrm{p}}(\%)$ with $N$ on the same $\log \varepsilon_{1 \mathrm{p}}(\%)-\log N$. The second summand describes the material phases in "range $\boldsymbol{B}$ and $\boldsymbol{C}$ " behaviour [10]. Werkmeister [10] made the model stress dependent as long as $A_{2}$ and $B_{2}$ were equal to zero in "range $A$ " stable behaviour.

Theyse also measured the accumulated permanent deformation produced in granular road bases using HVS tests. He proposed several non-linear functions [12,13] to model the accumulated permanent deformation of granular materials, obtaining good results. Table 1 presents one of these models (Equation 4) used by this researcher for materials under stable conditions. The model in question comprises two phases. First, an initial exponential deformation phase and second, a longterm linear increase rate in the permanent deformation. The model has an initial slope equal to the product of the two coefficients $A_{2}$ and $B_{2}$, a curvature determined by the value of coefficient $B_{2}$, an 
eventual linear slope equal to coefficient $m$, and, finally, an intercept with the Y-axis represented by coefficient $A_{2}$.

\section{Experimental methods and materials}

The unbound material tested was crushed granitic stone, frequently used as a base for road pavements in the region of Galicia (Spain). Its grading curve falls within the limits corresponding to the granular material designated as ZA25, defined in the "General technical specifications for works on roads and bridges" in Spain [1,14]. Laboratory samples measuring $100 \mathrm{~mm}$ in diameter and $200 \mathrm{~mm}$ in height were prepared using a special cylindrical mold. They were tested at the optimum moisture content and maximum dry density. Repeated load triaxial Constant Confining Pressure tests $(\mathbf{C C P})$ were carried out with a dynamic apparatus [1]. Eleven samples were subjected to different stress paths of $2 \times 10^{5}$ repeated load cycles with a sinusoidal wave frequency of $1 \mathrm{~Hz}$ (Figure 2).

\section{Verification of ranges of permanent deformation behaviour}

The stress paths were selected on the basis of the stresses supported by a well-designated, constructed section of pavement of low-traffic roads in use in Spain [1,15] (Figure 3). Therefore, according to the shakedown concept $[2,3]$ if the section is well designated, its structural behaviour should be in stable conditions, i.e., it should be within "range $\boldsymbol{A}$ " or, at the most, in "range $\boldsymbol{B}$ ".

In this way, figure 4 shows that in the samples with stress paths $\mathbf{P 1}, \mathbf{P 2}, \mathbf{P} \mathbf{3}$ and $\mathbf{P 6}$, the material is in "range A", since cumulative permanent axial strain undergoes a relatively minor increase, which would indicate that in practice, the stabilisation of the granular material has taken place. For stress path P11, the increase is a little more accentuated. It does not produce the stabilisation of permanent deformation, so the material exhibits a "range B" behaviour. As regards stress paths $\mathbf{P 4}$, $\mathbf{P 1 0}, \mathbf{P 7}, \mathbf{P 9}$ and $\mathbf{P 8}$, the material behaves as in "range $\mathbf{B}$ ". However, when $N$ increases it gives rise to an increment in permanent deformation. It is possible that if a higher $N$ were applied, the material might pass over to "range C". At the beginning of the test stress path P5 is in "range C", but, in the end, it exhibits the same behaviour as stress paths $\mathbf{P 4}, \mathbf{P 1 0}, \mathbf{P 7}, \mathbf{P 9}$ and $\mathbf{P 8}$. These results are considered quite acceptable since "range $C$ " would only be reached after substantial applications of traffic loads and the pavement is designated for low-traffic roads. 


\section{Proposed permanent deformation model}

As expressed by Pérez et al [1], equation 1 tends to underestimate the measured values while equation 2 tends to overestimate them, resulting in biased predictions. Therefore, for the purpose of improving the predictions, we decided to investigate the possibility of fitting another model. In this way, since equation 2 predicts underestimated values while equation 3 results in an overestimation, the objective was to join the two models in order to predict unbiased estimated values. The applicability of this model will be limited to low-traffic roads whose structural response behaviour, according to the shakedown concept, corresponds to "range $\boldsymbol{A}$ " and "range $\boldsymbol{B}$ ". The model in question (Equation 5, Table 1) also comprises two summands: The first summand is the Sweere model; the second is the Wolff model. As a result, it is made up of five parameters (Figure 5). However, as discussed earlier, the first term of equation 5 produces a linear increase of $\varepsilon_{1 \mathrm{p}}(\%)$ in relation to $N$ on a $\log \left(\varepsilon_{1 \mathrm{p}}\right)-\log (\mathrm{N})$ scale. It has a slope equal to $\infty$ at $N=0$ and equal to the product of the two coefficients $A_{1}$ and $B_{1}$ at $N=1$. After a certain number of load cycles, the second summand of equation 5 reproduces a linear increase of $\varepsilon_{1 \mathrm{p}}(\%)$ with $N$ on a $\varepsilon_{1 \mathrm{p}}-N$ scale. This increase is asymptotic to $(m \cdot N+A)$ at very high $N$ values. It has a slope equal to the product of the two coefficients $A_{2}$ and $B_{2}$ at $N=0$. The model has a curvature determined by the value of coefficients $B_{1}$ and $B_{2}$ and an eventual linear slope equal to coefficient $m$.

\section{Results and discussion}

Equations 3, 4 and 5 were selected for fitting in order to compare the functioning of the different models. The parameters were obtained by means of the Levenberg-Marquardt method [16]. The Levenberg-Marquardt algorithm interpolates between the Gauss-Newton algorithm and the method of gradient descent. It is more robust than the Gauss-Newton algorithm, which means that in many cases it finds a solution even if it starts far from the final minimum.

Table 2 shows all the values for the parameters of the eleven stress paths as well the determination coefficients $\mathbf{R}^{2}$ for equations 3, 4 and 5. Figure 6 reflects the values predicted for equation 3 and the measured data. The experimental data are very close to the continuous lines of the model. The adjustment is satisfactory, since the determination coefficients $\left(\mathbf{R}^{2}\right)$ yield a result of over $97.50 \%$ in all cases. Figure 7 presents the values predicted for equation 4 and the measured data. The 
experimental data are not as close to the continuous lines of the model as in equation 3 . The fit is not as good, as the $\mathbf{R}^{2}$ coefficients are only over $79.40 \%$ (Table 2). Figure 8 shows the values provided by equation 5 as well as the measured data. This fit is considered excellent, given that the $\mathbf{R}^{2}$ coefficients yield a result over $99.50 \%$ in all cases (Table 2 ).

In addition, the graphical results of these three models for the particular stress paths P8 (maximum permanent deformation) are reflected in figure 9. The fit of E3 is quite satisfactory, although some of the measured data do not match the model completely. It can be seen that 1PE3 is an increasing line with a positive curvature, showing much higher predicted values than the measured data, while 2PE3 is a decreasing line with a negative curvature until it stabilizes at $N=4 \times 10$ load cycles, predicting from this point a $\varepsilon_{1 \mathrm{p}}$ constant value equal to $-0.49 \%$. In other words, 2PE3 predicts negative values. This behaviour is similar in all of the other stress paths. Clearly, 2PE3 does not correctly describe the material response in "range $\boldsymbol{B}$ " as some researchers have argued $[10,11]$.

In this figure it is also possible to observe that the values predicted by 1PE4 and 2PE4 are lower than the measured values. Moreover, 1PE4 is a straight line that intercepts the Y-axis in zero; while 2PE4 has an initial curvature until it stabilizes, predicting a $\varepsilon_{1 \mathrm{p}}$ constant value equal to $1.81 \%$ starting at approximately 100 load cycles. It is evident that that E4 does not provide a very satisfactory fit to the model. This performance is similar in all the other stress paths. Obviously, and as contended by Theyse [12,13], E4 does not accurately describe the material response in "range A".

Figure 9 highlights a very close fit between the predicted values with equation 5 and the measured values. Undoubtedly, this model adjustment is much better than the fits of equation 3 and equation 4. This figure also displays the values predicted for 1PE5 and 2PE5, separately. Both predictions are below the measured values. It can be seen that starting at approximately $1 \times 10^{3}$ load cycles, the values predicted for the asymptote are the same as those predicted for 2PE5. In the figure it is also possible to see that the values predicted for 1PE5 are higher than those predicted for 2PE5. E5 clearly provides an excellent fit as the measured values match the model almost perfectly. It is only natural that the fit to the data would improve when the number of parameters in the model is increased. Actually, E5 includes Sweere's and Wolff's models as particular cases. However, the 
resulting accuracy proved to be remarkable even when this effect is taken into account, and it is well worth having to manipulate a few more parameters. By examining each term's contribution to the fitting curves, it becomes apparent that Sweere's power-like term governs the behaviour of the solution for relatively small values of $N$, while Woll's perturbed linear term gradually acquires relevance as the number of load cycles increases, eventually giving the curve an asymptotic slope. Finally, if a rut depth is estimated as the product of $\varepsilon_{1 \mathrm{p}}$ and the thickness of the granular material, then again, in figure 10 the rut predictions estimated by means of equations 3 and 4 are not close to the values calculated as the product of the measured strain and $200 \mathrm{~mm}$. It is also demonstrated that equations 3 and 4 tend to underestimate the calculated values for a large number of cycles, whereas equation 5 does not. On the contrary, equation 5 tends neither to underestimate or overestimate the values.

\section{Conclusion}

Equation 3 offers biased predictions since it tends to underestimate the measured values. Moreover, the second summand of this model does not provide a satisfactory description of the material behaviour in "range $\boldsymbol{B}$ " of the shakedown concept.

Equation 4 offers biased predictions as it also underestimates the measured values. It has been demonstrated that this model does not accurately describe the material behaviour in "range A".

Equation 5 offers excellent predictions since it neither underestimates nor overestimates the measured values. Hence, equations 1 and 2 are models that work much better as a single unit than separately. It has been proven that this model offers an excellent description of the material behaviour in "range $\boldsymbol{A}$ and $\boldsymbol{B}$ " in a pavement section of low-traffic roads.

Finally, it is important to clarify that this paper presents merely a preliminary approach to the problem. Hence, future research will require systematic procedures using a higher number of stress paths. Also to be taken into account is that these results are based on repeated load triaxial (RLT) permanent deformation tests and not on tests with actual road pavements.

\section{Acknowledgments}

The authors would like to thank Xavier Dominguez for his assistance with data analysis. 


\section{References}

[1] Pérez I, Medina L, Romana MG. Permanent deformation models for a granular material used in road pavements. Construction and Building Materials 2006, 20: 790-800.

[2] Werkmeister S, Dawson A, Wellner F. Permanent deformation behaviour of granular materials and the shakedown concept. Transportation Research Record 2001; 1757: 75-81.

[3] Werkmeister S, Dawson A, Wellner, F. Pavement design model for unbound granular materials. Journal of Transportation Engineering 2004; 130: 665-674.

[4] Sweere GTH. Unbound granular bases for roads. Ph.D. Thesis, University of Delft, 1990.

[5] Wolff H, Visser A. T. Incorporating elasto-plasticity in granular layer pavement design. In Proceedings of the Institution of Civil Engineers Transport 1994; 105: 259-272.

[6] Theyse H L. Mechanistic-empirical modelling of the permanent deformation of unbound pavement layers. In: ISAP $8^{\text {th }}$ International Conference on Asphalt Pavement. Seattle (USA), 1997.

[7] Francken L, Clauwaert C. Characterization and structural assessment of bound materials for flexible road structures. In: Proceedings of the $6^{\text {th }}$ International Conference on the Structural Design of Asphalt Pavements. Ann Arbor, Michigan, 1987, p. 130-144.

[8] Kaloush K, Witczak MW. Tertiary flow characteristics of asphalt mixtures. Journal of Asphalt Paving Technology, 2002; 71: 248-280

[9] Huurman M. Rut development in concrete block pavements due to permanent strain in the substructure. In: First International Conference on Concrete Block Paving. Pave Israel 96, 1996, p 293-303.

[10] Werkmeister S. Permanent deformation behaviour of unbound granular materials in pavement construction. PhD thesis. Dresden University of Technology, Germany, 2003

[11] Arnold G. Rutting of Granular Pavement. PhD thesis. University Of Nottingham, England. UK, 2004.

[12] Theyse HL. The development of mechanistic-empirical permanent deformation design models for unbound pavement materials from laboratory accelerated pavement data. Proceedings of the fifth international symposium on unbound aggregates in road, UNBAR 5, Nottingham, United Kingdom, 2000, p. 285-293.

[13] Theyse HL. Stiffeness, Strenght, and Performance of Unbound Aggregate Material: Application of South African HVS and Laboratory Results to California Flexible Pavements. University of California Pavement Research Center, 2002. p. 1-86.

[14] Ministerio de Fomento. Anexo actualización PG-3. Capas estructurales de firmes. Anexo del pliego de prescripciones técnicas generales para obras de carreteras y puentes. In: Bustos; G., editor. PG-3 Anexo de actualización. Ediciones Liteam, 2002. p. 1-15.

[15] Ministerio de Fomento. Secciones de firme de la instrucción de carreteras. In: Bustos; G., editor. 6.1-IC Texto Oficial. Madrid: Ediciones Liteam, 2002. p. 1-22.

[16] Marquardt DW. An algorithm for least-squares estimation of nonlinear parameters. Journal of the Society for Industrial and Applied Mathematics 1963, 11: 431-441. 


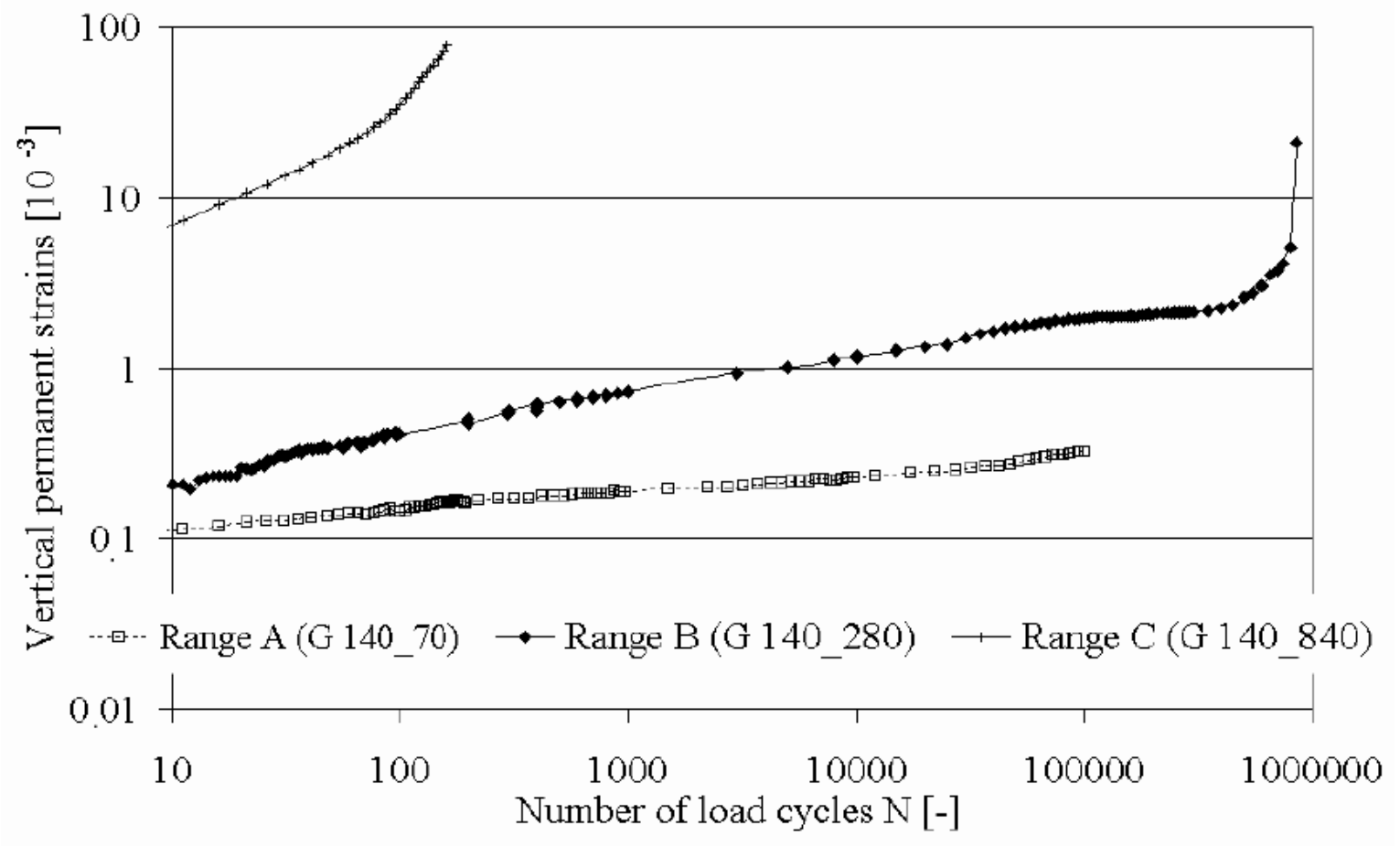

Figure 1. Typical vertical permanent strain versus number of load cycles ( $\mathrm{G} n \_\mathrm{m}=\boldsymbol{G}$ ranodiorite at a cell pressure of $\boldsymbol{n} \mathrm{kPa}$ and deviator stress of $\boldsymbol{m} \mathrm{kPa}$ ) [5] 


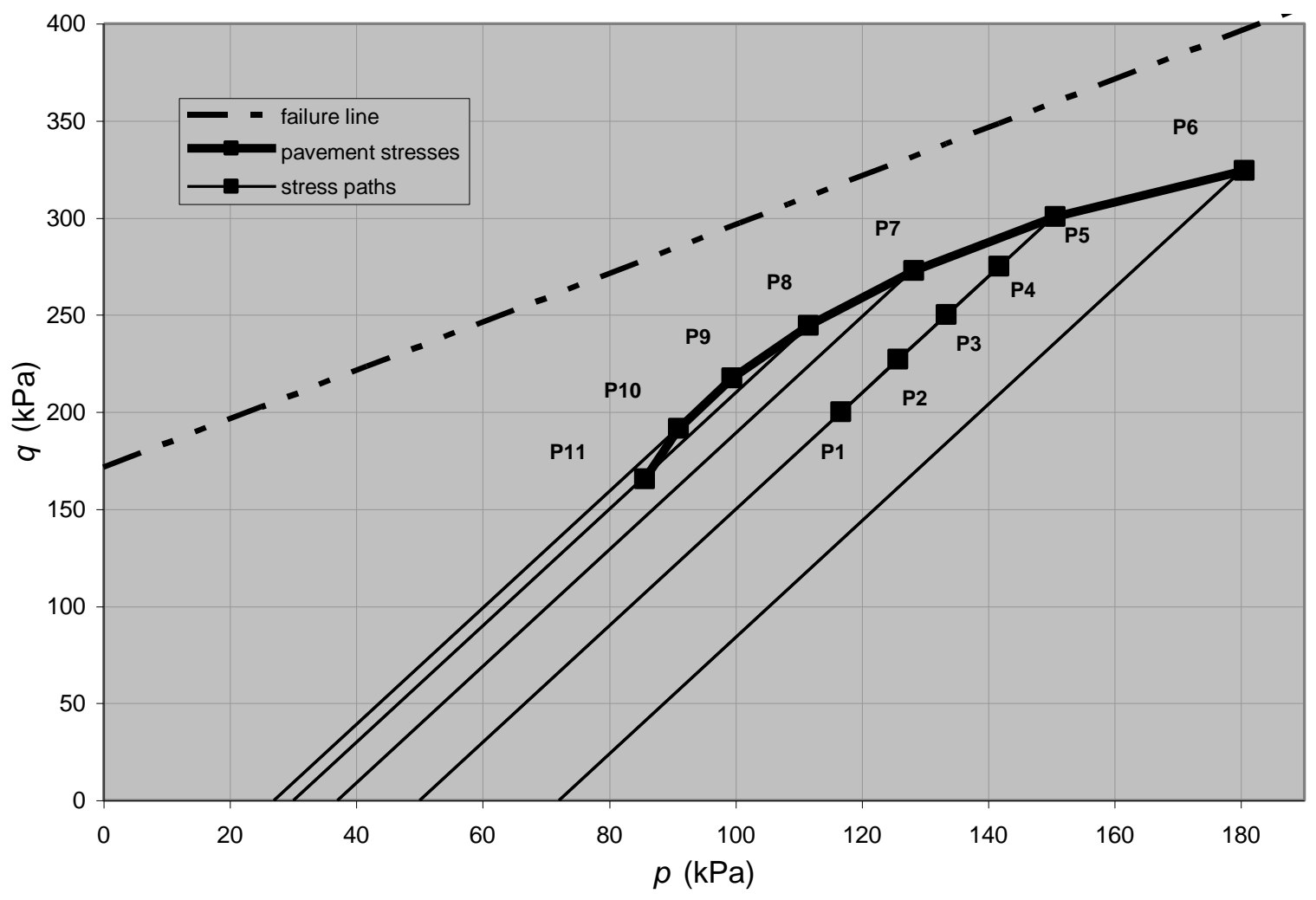

Figure 2. Stress Paths. 


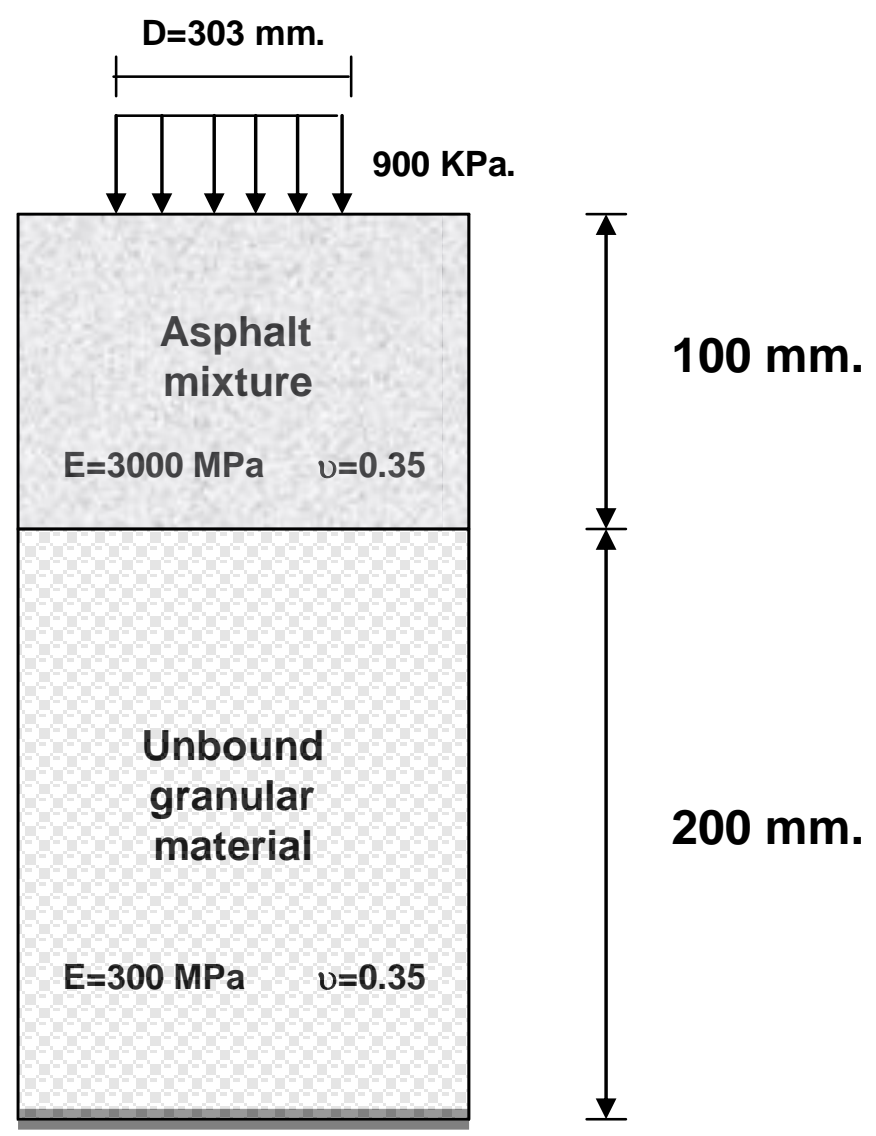

\section{Cement Stabilised} soil

Figure 3. Pavement section. 


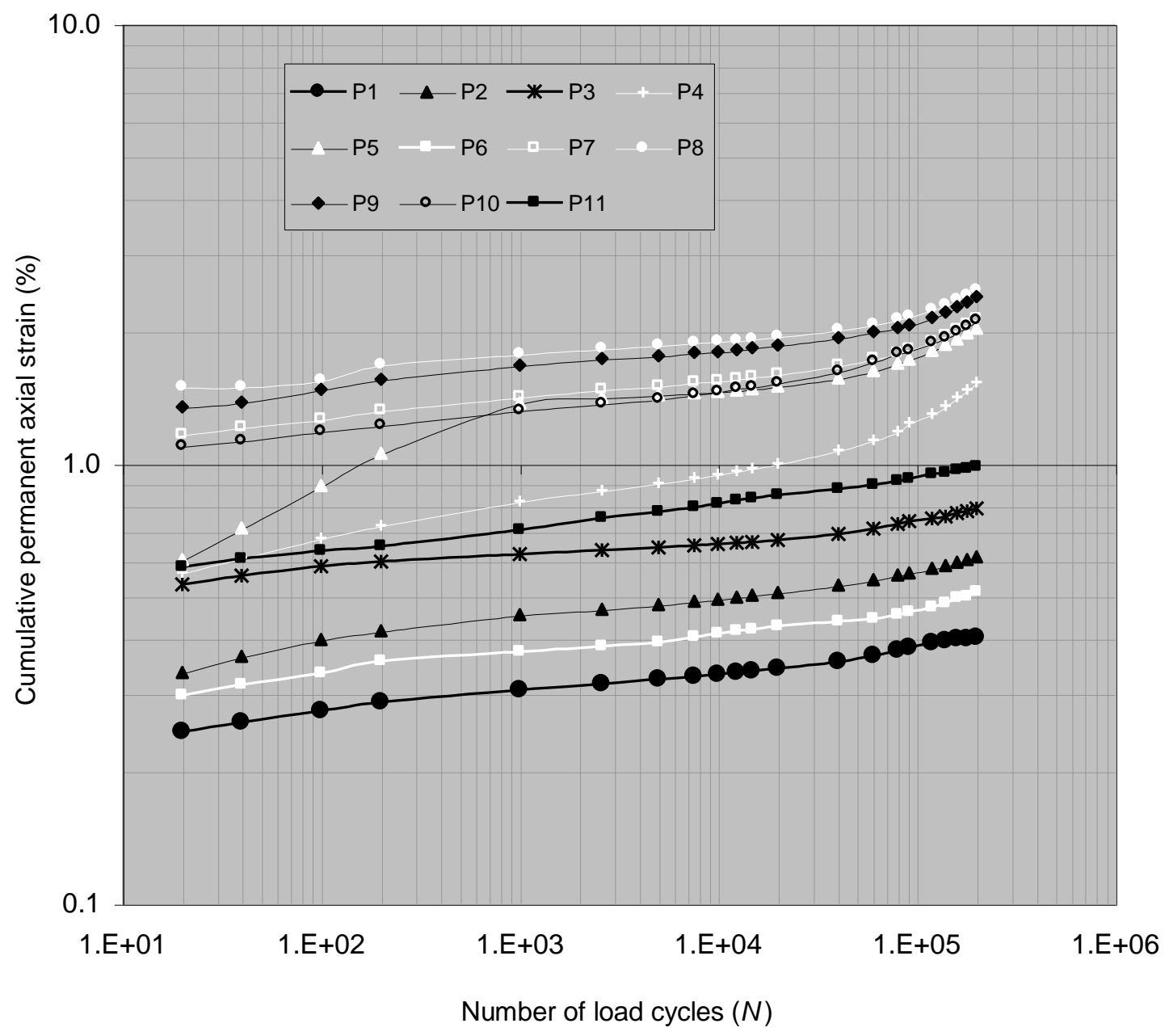

Figure 4. Permanent deformation measured versus load cycles of stress paths. 


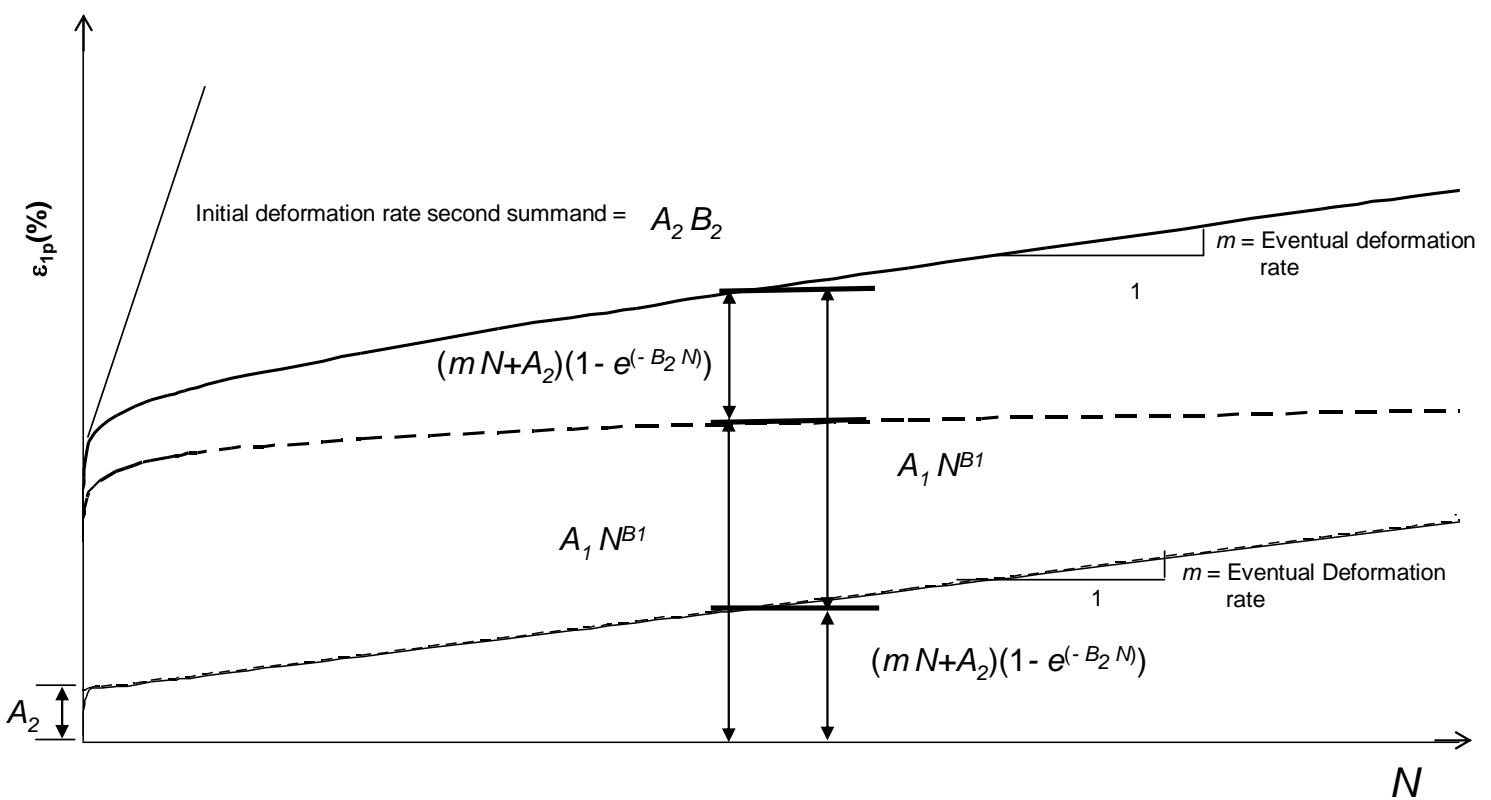

Figure 5. Equation 5 parameters. 


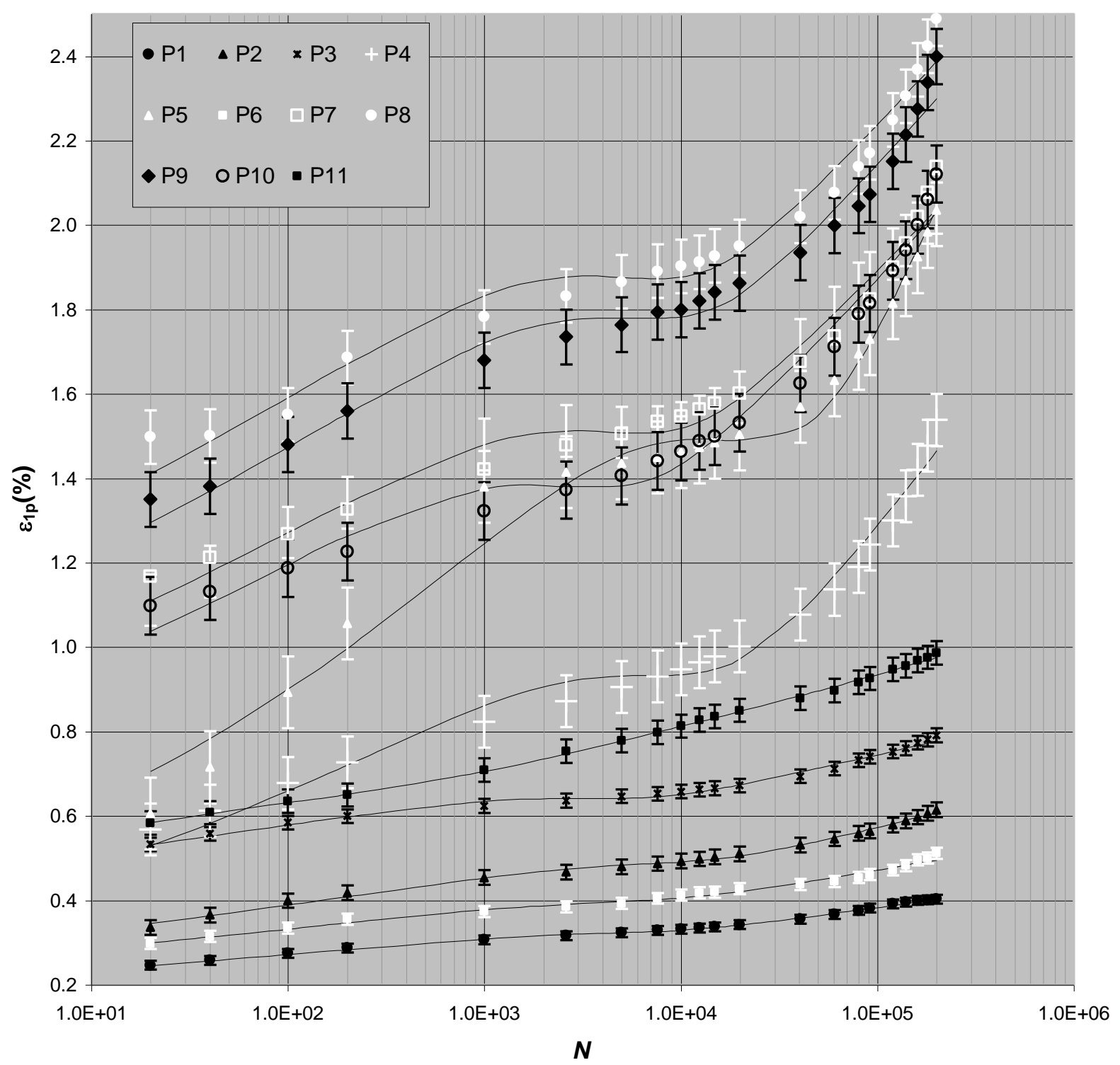

Figure 6. Permanent deformation versus load cycles (with equation 3) Standard error bars represent the $99 \%$ confidence interval of a mean 


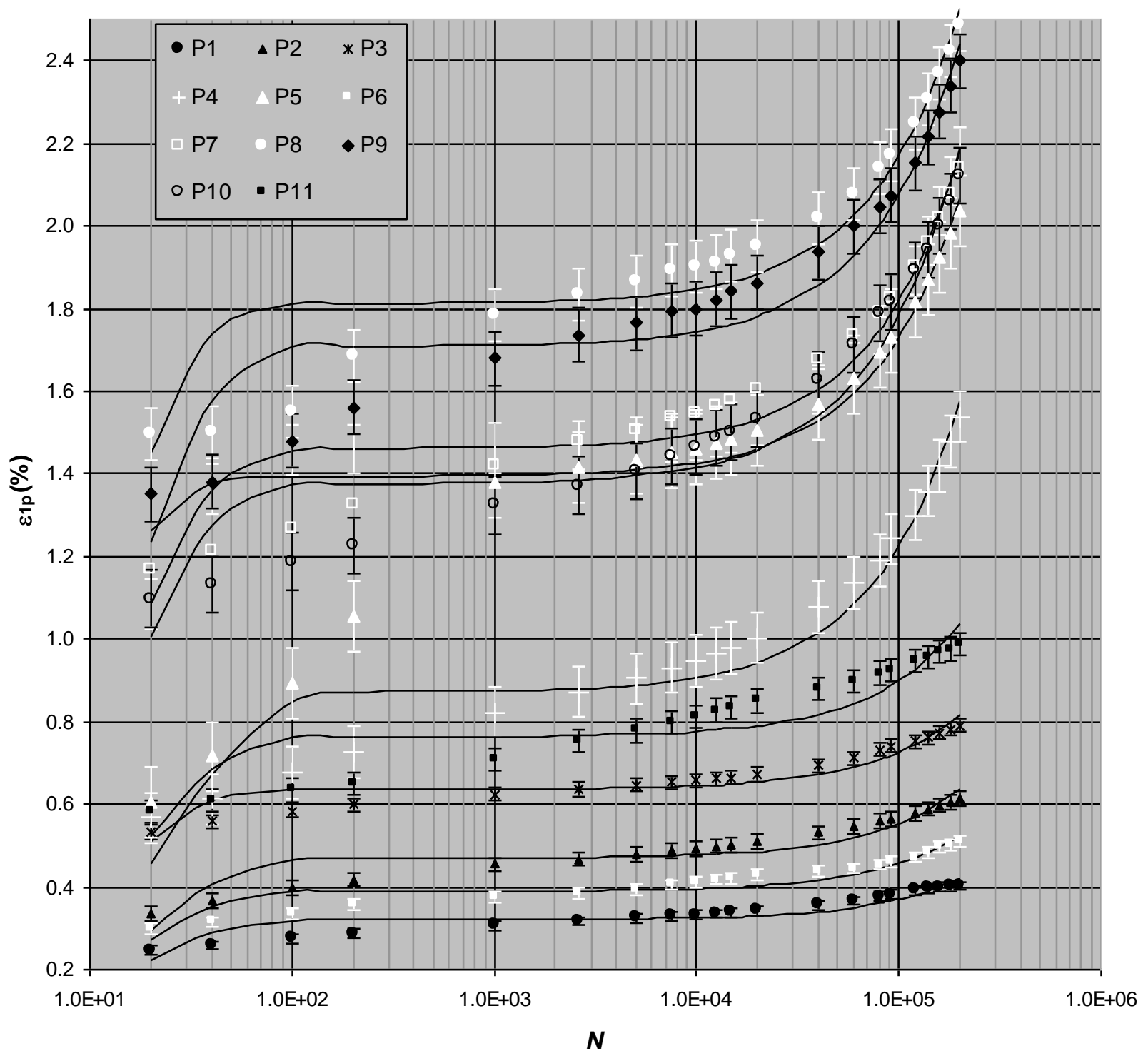

Figure 7. Permanent deformation versus load cycles (with equation 4)

Standard error bars represent the $99 \%$ confidence interval of a mean 


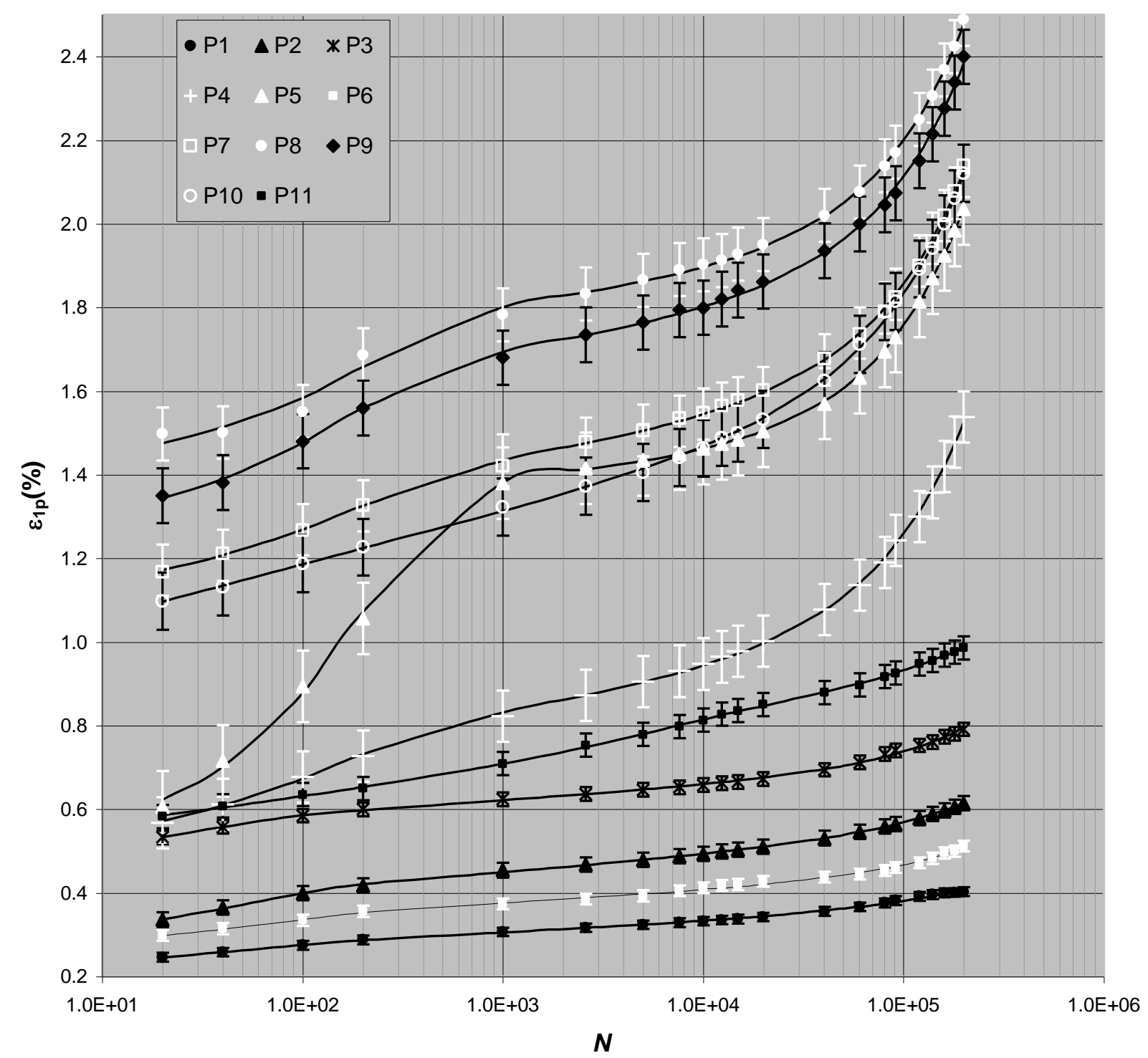

Figure 8. Permanent deformation versus load cycles (with equation 5) Standard error bars represent the 99\% confidence interval of a mean 


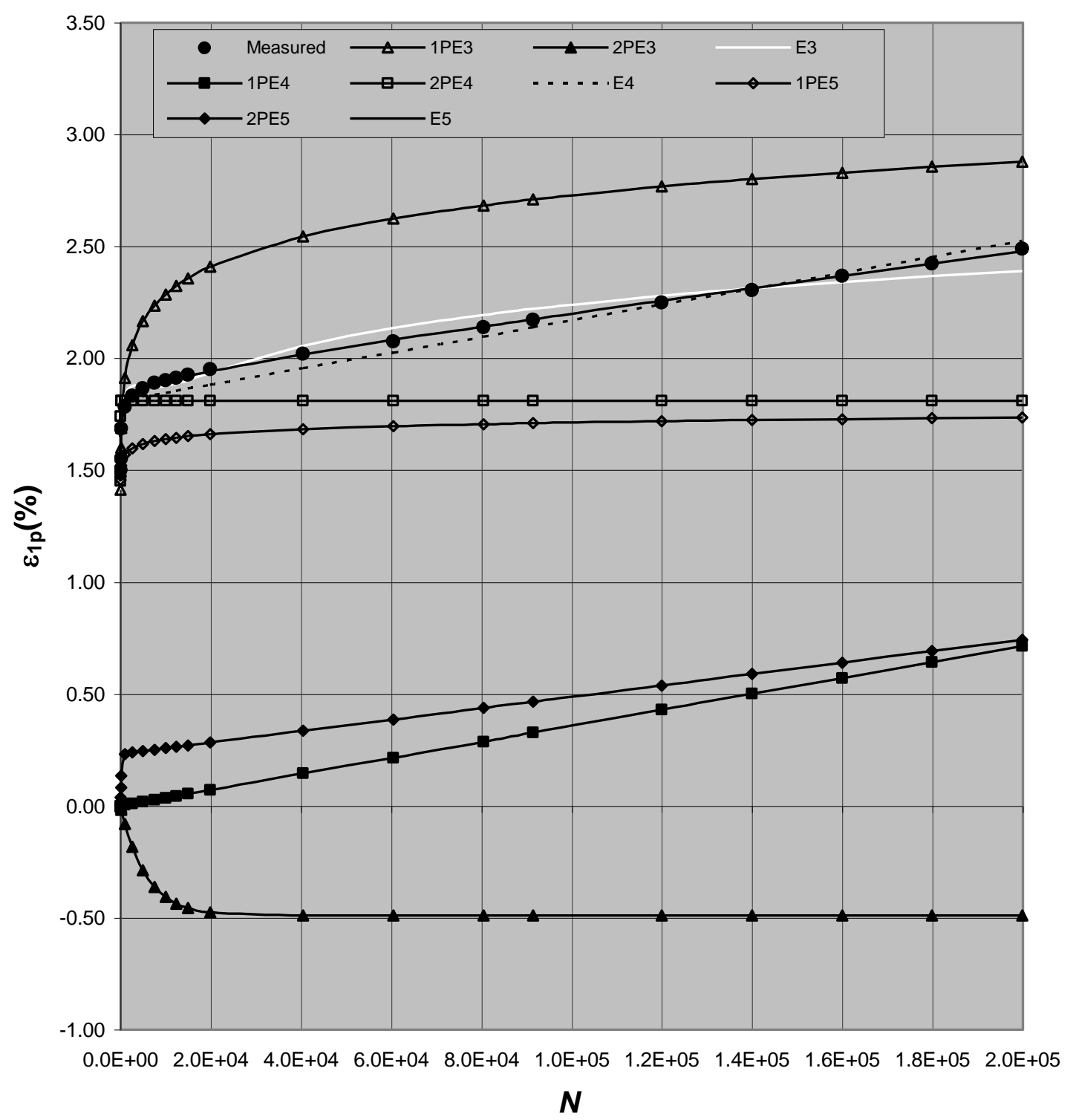

E3=Equation 3; 1PE3= First part equation 3; 2PE3=Second part equation 3

E4=Equation 4; 1PE4= First part equation 4; 2PE4=Second part equation 4

$\mathrm{E} 5=$ Equation $5 ; 1 \mathrm{PE} 5=$ First part equation $5 ; 2 \mathrm{PE} 5=$ Second part equation 5

Figure 9. Permanent deformation versus load cycles (stress path P8) 

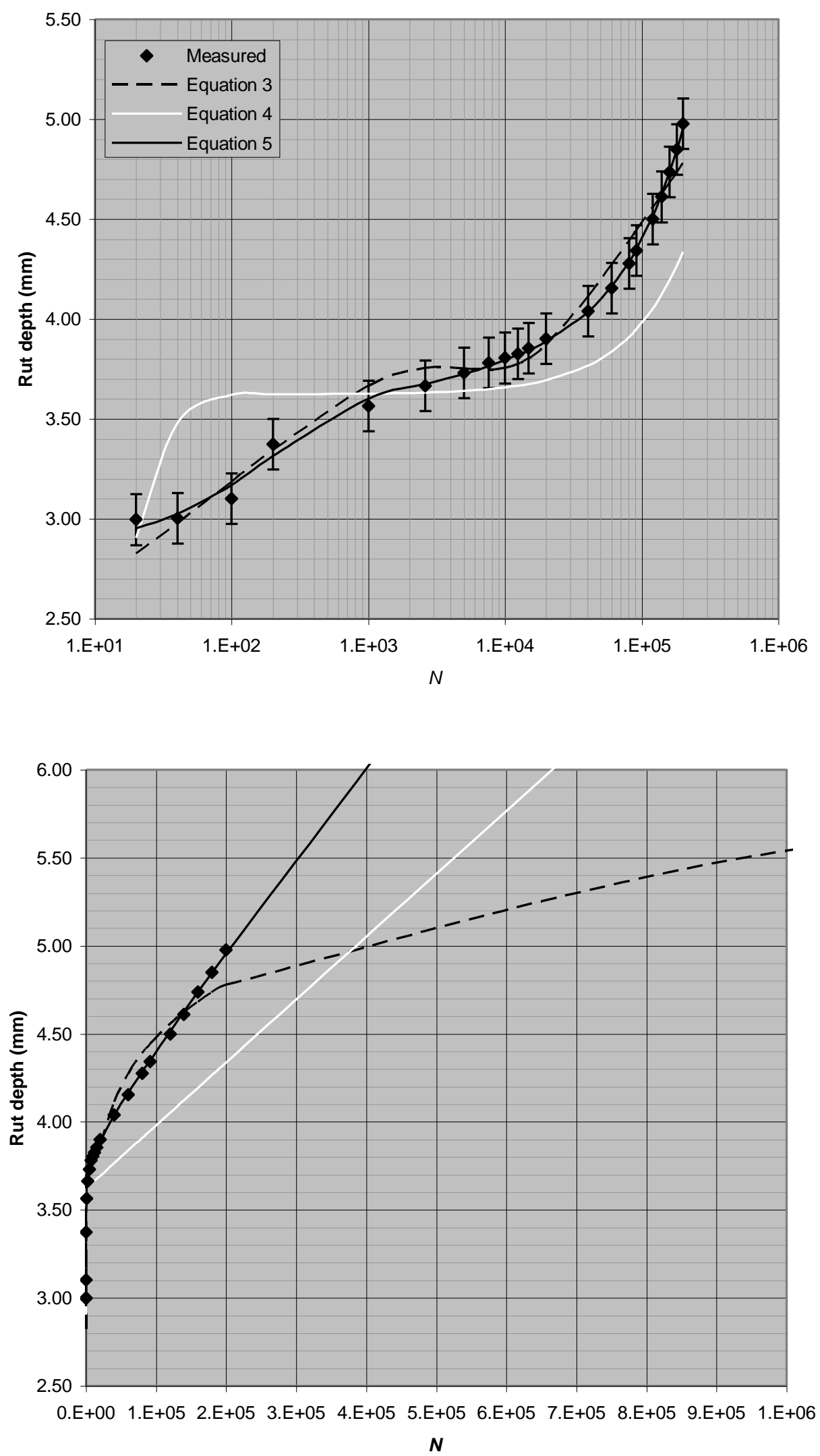

Figure 10. Prediction of rut depths

Standard error bars represent the $99 \%$ confidence interval of a mean 
Table 1. Permanent deformation models

\begin{tabular}{|c|c|l|l|}
\hline \multicolumn{5}{|c|}{ Models linking permanent deformation to the number of load cycles } \\
\hline Equation & \multicolumn{1}{|c|}{ Model } & \multicolumn{1}{|c|}{ Reference } & \multicolumn{1}{c|}{$\begin{array}{l}\text { Regression } \\
\text { parameters }\end{array}$} \\
\hline 1 & \multicolumn{1}{|c|}{$\varepsilon_{1 p}(\%)=A \cdot N^{B}$} & Sweere [4] & $A, B$ \\
\hline 2 & $\varepsilon_{1 p}(\%)=(m \cdot N+A)\left(1-e^{-B \cdot N}\right)$ & Wolff et al [5] & $A, B, m$ \\
\hline 3 & $\varepsilon_{1 p}(\%)=A_{1} \cdot N^{B_{1}}+\left(A_{2}\right)\left(e^{-B_{2} \cdot N}-1\right)$ & $\begin{array}{l}\text { Francken et al [7], Kaloush } \\
\text { et al [8], Huurman [9], } \\
\text { Werkmeister [10], Arnold } \\
{[11]}\end{array}$ & $A_{1}, B_{1}, A_{2}, B_{2}$ \\
\hline 4 & $\varepsilon_{1 p}(\%)=(m \cdot N)+\left(A_{2}\right)\left(1-e^{-B_{2} \cdot N}\right)$ & Theyse [12,13] & $A_{2}, B_{2}, m$ \\
\hline 5 & $\varepsilon_{1 p}(\%)=A_{1} \cdot N^{B_{1}}+\left(m \cdot N+A_{2}\right)\left(1-e^{\left.-B_{2} \cdot N\right)}\right.$ & - ----- & $\begin{array}{l}A_{1}, B_{1}, m, A_{2}, \\
B_{2}\end{array}$ \\
\hline
\end{tabular}


Table 2. Model parameters

\begin{tabular}{|c|c|c|c|c|c|c|c|c|c|c|c|c|c|c|c|c|c|c|c|c|c|}
\hline \multirow{2}{*}{\multicolumn{4}{|c|}{ Parameter }} & \multicolumn{3}{|c|}{$A_{1}$} & \multicolumn{3}{|c|}{$B_{1}$} & \multicolumn{3}{|c|}{$m$} & \multicolumn{3}{|c|}{$A_{2}$} & \multicolumn{3}{|c|}{$B_{2}$} & \multicolumn{3}{|c|}{$R_{2}$} \\
\hline & & & & \multicolumn{3}{|c|}{ Equation } & \multicolumn{3}{|c|}{ Equation } & \multicolumn{3}{|c|}{ Equation } & \multicolumn{3}{|c|}{ Equation } & \multicolumn{3}{|c|}{ Equation } & \multicolumn{3}{|c|}{ Equation } \\
\hline Stress Path & $q(\mathrm{kPa})$ & $p(\mathrm{kPa})$ & $\sigma_{3}(\mathrm{kPa})$ & 3 & 4 & 5 & 3 & 4 & 5 & 3 & 4 & 5 & 3 & 4 & 5 & 3 & 4 & 5 & 3 & 4 & 5 \\
\hline $\mathrm{P} 1$ & 200.00 & 116.67 & 50.00 & 0.2028 & ---- & 0.210 & 0.0647 & --- & 0.041 & --- & $5.087 \times 10^{-7}$ & $1.842 \times 10^{-7}$ & 0.0433 & 0.320 & 0.025 & 0.0002061 & -0.059 & -0.018 & 99.51 & 86.03 & 99.70 \\
\hline $\mathrm{P} 2$ & 227.00 & 125.66 & 50.00 & 0.2773 & --- & 0.275 & 0.0745 & --- & 0.045 & --- & $8.445 \times 10^{-7}$ & $3.149 \times 10^{-7}$ & 0.0800 & 0.469 & 0.071 & 0.0001327 & -0.050 & -0.017 & 99.57 & 87.50 & 99.97 \\
\hline P3 & 250.00 & 133.33 & 50.00 & 0.4535 & --- & 0.465 & 0.0544 & --- & 0.026 & --- & $8.935 \times 10^{-7}$ & $4.522 \times 10^{-7}$ & 0.1025 & 0.636 & 0.062 & 0.0002870 & -0.081 & -0.031 & 99.25 & 89.46 & 99.83 \\
\hline $\mathrm{P} 4$ & 275.00 & 141.66 & 50.00 & 0.3505 & --- & 0.472 & 0.1394 & --- & 0.054 & --- & $3.535 \times 10^{-6}$ & $2.337 \times 10^{-6}$ & 0.4546 & 0.871 & 0.142 & 0.0001295 & -0.037 & -0.006 & 98.63 & 94.02 & 99.94 \\
\hline P5 & 300.00 & 150.00 & 50.00 & 0.4463 & --- & 0.446 & 0.1536 & --- & 0.153 & --- & $3.374 \times 10^{-6}$ & $2.651 \times 10^{-6}$ & 0.8665 & 1.392 & 0.753 & 0.00005067 & -0.119 & -0.004 & 98.52 & 98.03 & 99.96 \\
\hline P6 & 324.00 & 180.00 & 72.00 & 0.2480 & --- & 0.258 & 0.0646 & -- & 0.037 & --- & $6.785 \times 10^{-7}$ & $2.927 \times 10^{-7}$ & 0.0493 & 0.389 & 0.041 & 0.0002081 & -0.060 & -0.013 & 98.52 & 86.99 & 99.73 \\
\hline P7 & 273.00 & 128.00 & 37.00 & 0.8569 & --- & 1.036 & 0.0874 & -- & 0.028 & --- & $3.610 \times 10^{-6}$ & $2.411 \times 10^{-6}$ & 0.4498 & 1.459 & 0.025 & 0.0002135 & -0.068 & -0.005 & 97.60 & 92.49 & 99.94 \\
\hline P8 & 245.00 & 112.00 & 30.00 & 1.1221 & --- & 1.375 & 0.0772 & -- & 0.019 & --- & $3.577 \times 10^{-6}$ & $2.555 \times 10^{-6}$ & 0.4890 & 1.811 & 0.071 & 0.0001785 & -0.081 & -0.004 & 97.52 & 92.69 & 99.80 \\
\hline P9 & 217.00 & 99.00 & 27.00 & 1.0133 & --- & 1.224 & 0.0827 & --- & 0.024 & --- & $3.667 \times 10^{-6}$ & $2.430 \times 10^{-6}$ & 0.4794 & 1.709 & 0.062 & 0.0001645 & -0.064 & -0.005 & 98.16 & 90.62 & 99.91 \\
\hline P10 & 191.00 & 91.00 & 27.00 & 0.7842 & --- & 0.957 & 0.0945 & --- & 0.042 & --- & $4.083 \times 10^{-6}$ & $2.391 \times 10^{-6}$ & 0.4532 & 1.375 & 0.142 & 0.0003417 & -0.066 & -0.002 & 98.29 & 92.68 & 99.95 \\
\hline P11 & 165.00 & 85.00 & 30.00 & 0.4930 & --- & 0.509 & 0.0588 & --- & 0.046 & --- & $1.365 \times 10^{-6}$ & $2.623 \times 10^{-6}$ & 0.0341 & 0.763 & 0.753 & 0.0049900 & -0.058 & -0.000 & 99.86 & 79.42 & 99.96 \\
\hline
\end{tabular}

